\title{
Defect and transmission properties of two-dimensional quasiperiodic photonic band-gap systems
}

\author{
Samuel S. M. Cheng, Lie-Ming Li, C. T. Chan, and Z. Q. Zhang \\ Department of Physics, Hong Kong University of Science and Technology, Clear Water Bay, Hong Kong, China
}

(Received 17 August 1998)

\begin{abstract}
It has recently been demonstrated that two-dimensional photonic band gaps can be realized in systems comprising of a quasiperiodic arrangement of dielectric cylinders. We show that waveguides crafted out of such photonic "quasicrystals" can be used to guide light around sharp corners, just as in the case of periodic photonic band-gap systems, but the quasiperiodic systems tend to be more frequency selective. Because of the absence of translational symmetry, these quasiperiodic photonic band-gap structures also display richer defect properties. Spectral gaps for both the TM and TE polarizations in the same frequency range can be realized in metallodielectric configurations. [S0163-1829(99)10505-8]
\end{abstract}

\section{INTRODUCTION}

Photonic band-gap (PBG) material has received a lot of attention in recent years. ${ }^{1,2}$ From a material point of view, PBG material is a remarkable example of material by design. It is a new class of composite material which does not exist in nature, but is conceived, designed, and made by man. The existence of a spectral gap in the electromagnetic (EM) wave spectrum opens up many exciting possibilities, such as the suppression of spontaneous emission, ${ }^{3}$ the possible observation of interesting quantum interference effects, ${ }^{4}$ and the realization of strong photon localization. ${ }^{5}$ Many potential applications including quantum electronic devices, distributedfeedback mirror, light-emitting diodes, ${ }^{6}$ high- $Q$ cavities, ${ }^{7}$ microwave antennae substrate, ${ }^{8}$ and optical waveguides ${ }^{2,9}$ have further fueled the interest in PBG research.

PBG materials are frequently viewed as photonic analogs of electronic semiconductors, and the term "photonic crystal" has been coined and used in many occasions to represent manmade inhomogeneous material with a periodic texture that exhibits photonic gaps. For electronic materials, the research activities have extended progressively from the study of the perfect crystal to the properties of defects, surfaces, and eventually to the noncrystalline (such as quasiperiodic and amorphous) environment. For the case of bulk photonic crystals, the structure and the related optical properties have been studied both in two and three dimensions, although new ideas to improve photonic gaps are still emerging. ${ }^{10}$ The properties of defects and surface states have been considered and well documented, ${ }^{2}$ and these studies already represent a step beyond crystallinity, but the considerations are still based on the periodicity of a perfect photonic crystal. It would be interesting to take one step further and investigate what happens to the photonic gap if we do away with the periodicity. Is the photonic gap still there, and if so, how would the overall properties be affected? Recently, it has been shown that photonic gaps can be found in a two-dimensional (2D) quasiperiodic arrangement of dielectric cylinders, demonstrating the existence of photonic gaps without periodicity and translational symmetry. ${ }^{11}$ The purpose of this paper is to examine in greater detail the property of such quasiperiodic photonic band-gap systems when "point" defects are introduced to make microcavities and when "line" defects are introduced to create light guides. We found that the behavior is in general more complex than periodic PBG materials. We also examine the possibility of using metallodielectric materials to facilitate the formation of photonic gaps common to both the TM and TE polarizations.

\section{METHOD OF CALCULATION}

In this paper, we will study the defect properties and the transmission and scattering properties of waveguides derived from quasiperiodic PBG structures. The two computational methods employed are already described in detail elsewhere, ${ }^{12,13}$ and we will only provide a brief description in the following.

The existence of spectral gaps and the defect properties of quasiperiodic photonic materials are studied with a "finitedifference-time-domain" method. ${ }^{12}$ The time evolution of the fields as governed by the Maxwell equations are solved on a uniform grid in both the spatial and the time domain. When the boundary conditions are given and the fields are specified at any instant $t=0$, the spatial derivatives, and hence the "curls" of the fields can be determined using finite differences. The Maxwell equations then give the time derivatives of the fields which allow us to update the $H(r, t)$ and $E(r, t)$ fields in the time domain. The fields $H(r, t)$ and $E(r, t)$ can then be recorded as a time series for some sampling points in the system, and for a sufficiently large number of time steps (which governs the resolution in the frequency domain), the time series are Laplace transformed to the frequency domain to obtain the spectral intensities. The intensity at a particular frequency depends on the projection of the initial field onto the normal modes of that frequency. The initial field intensities are chosen to be random numbers, and the corresponding spectral intensities correspond to the density of states. Thus, the existence of a photonic gap can be found rather straightforwardly. This method is an "order $(N)$ " method, in the sense that the computational effort scales linearly with the number of grid points, which itself scales linearly with the system size. In contrast, the computational effort of band-theoretic techniques like the popular plane-wave method ${ }^{14}$ generally scales like the third power of size of the system. This method was conceived and derived from our previous experience with molecular dynamics, 


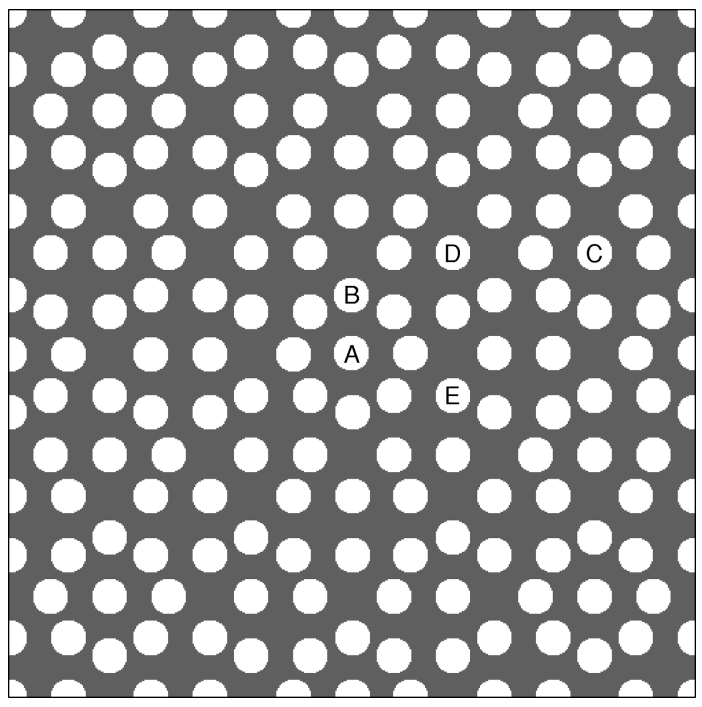

FIG. 1. Schematic figure showing the octagonal quasiperiodic arrangement of cylinders. The cylinders occupy a volume fraction of $30 \%$ and have $\epsilon=10$ and the background has $\epsilon=1$.

where velocity-velocity correlation functions can give phonon normal-mode frequencies. When adapted to EM waves, we found that the approach is essentially the same as the finite-difference-time-domain method that was very popular in the engineering field. ${ }^{15}$

The transmission properties are studied with a multiplescattering formulation, ${ }^{13,16}$ which is best suited for a finite collection of cylinders with a continuous incident wave of fixed frequency. For circular cylinders, the scattering property of the individual cylinder can be obtained analytically, ${ }^{17}$ relating the scattered fields to the incident fields. The total field, which includes the incident plus the multiple-scattered field, can then be obtained by solving a linear system of equations, whose size is proportional to the number of cylinders in the system. Both near field and far field radiation patterns can be obtained straightforwardly. Such an approach is a very efficient way of handling the scattering problem of a finite sample containing cylinders of circular cross sections (or spheres in three dimensions) in an arbitrary arrangement.

\section{RESULTS}

Before we go into the numerical results, we discuss briefly the octagonal quasiperiodic structure itself. ${ }^{18}$ It is formed by placing dielectric cylinders with circular cross sections in the vertices of a two-dimensional octagonal quasiperiodic lattice. The cylinders have $\epsilon=10$ and occupy a volume fraction of $30 \%$ in a background of $\epsilon=1$. The $2 \mathrm{D}$ octagonal quasiperiodic pattern is tiled by squares and rhombuses (with an acute angle of $45^{\circ}$ ) of equal side length $a$. Unless otherwise specified, all angular frequencies referred to in this paper are measured in units of $2 \pi(c / a)$, where $c$ is the speed of light and $a$ is the length of a side of the square or the rhombus ${ }^{19}$ in the octagonal tiling. The arrangement of the cylinders is shown schematically in Fig. 1, and the positions marked A, B, C, D, and E are some sites from which cylinders will be removed in order to study defect states. Such structures give rise to spectral gaps in TM polarized modes. In this paper, TM mode refers to modes with the $E$

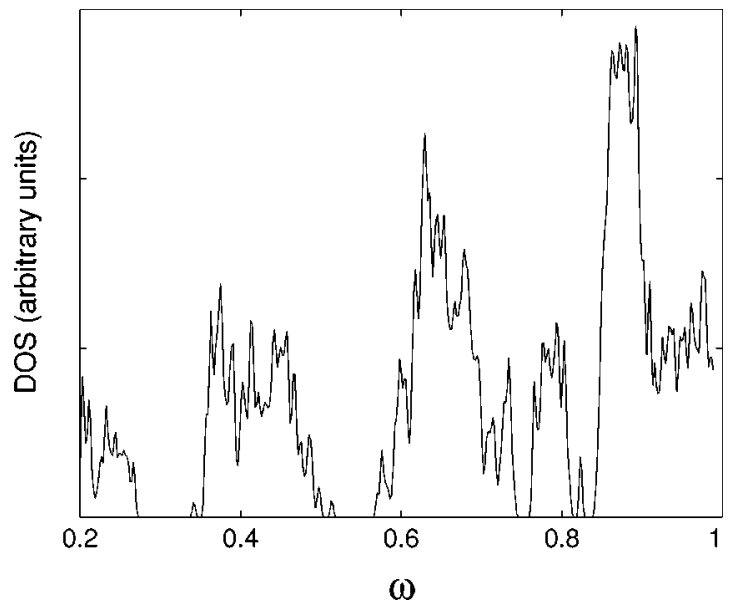

FIG. 2. TM mode density of states for a missing cylinder at position $A$ (see Fig. 1).

field aligned along the axis of the cylinders and the magnetic fields are in the 2D plane, while TE modes refer to modes with their $H$ field along the axis of the cylinders and the $E$ field in the 2D plane.

\section{A. Defect properties}

In the density of states calculations, the finite difference algorithm requires a specific boundary condition. We found that it is most convenient to impose the periodic boundary conditions on a square sample. Most of the calculations are done with a sample containing 164 cylinders, discretized on a $616 \times 616$ grid. We typically use $2 \times 10^{5}$ time steps in the simulation, with each time step of the order of $10^{-3}(\mathrm{a} / \mathrm{c})$. Since the periodic boundary condition is imposed, the fields can be written in the Bloch form. The results reported below are calculated with sampling at the $k=0$ Bloch momentum. Since our "supercell" is large, this sampling is adequate except for frequencies lower than $\omega=0.15$. At low frequencies (below $\omega=0.15$ ), the calculated DOS become a collection of isolated peaks and the gaps in between are artifacts of this finite sampling due to the low density of modes at low frequencies. For $\omega<1$, we observe four major spectral gaps at about $\omega=0.3,0.55,0.75$, and 0.82 . These gaps are the consequences of the quasiperiodic arrangement of the cylinders, and has nothing to do with artificial periodic boundary condition we imposed in the simulation. The "supercell" we used in the simulation is reasonably large, and if the gaps are an artifact of the periodicity of the supercell, the lowest gap should appear at a much lower frequency than the first spectral gap shown in the following density-of-state plots (see, for example, Fig. 2). This point has already been established earlier. ${ }^{11}$

In 2D PBG systems consisting of an array of cylinders, a structural defect can be formed simply by the removal or the displacement of one cylinder. Such a structural defect (the missing cylinders) can be regarded as a microcavity and it is well established that spatially localized defect modes can be formed. If we have a periodic PBG system to start with, the removal of one single cylinder will create one fixed set of defect states. Unlike periodic systems, the quasiperiodic arrangement does not have translational symmetry and the cylinders have different local environments. Thus, the proper- 
ties of the defect states depend on which cylinder is being removed, leading to a large variety of possibilities. To illustrate this point, we first remove one cylinder at site $A$ (see Fig. 1), which is surrounded by eight nearest-neighbor cylinders. The corresponding TM mode density of states are shown in Fig. 2, and defect states appear as isolated peaks in the spectral gaps. We find defect states in the first, second, and fourth gaps at about $\omega=0.34,0.51$, and 0.82 , respectively. The electric field intensities $\left(E_{z}^{2}\right)$ of these modes projected onto a 2D plane for these modes are shown in the three panels in Fig. 3, respectively. Since these defect states have frequencies that are forbidden in the quasiperiodic lattice, they have to be localized around the defect site, which is precisely what is observed in the figures. We now remove a cylinder at site $B$ (see Fig. 1). The corresponding DOS for the system with this defect is shown in Fig. 4, which shows a defect state in the second gap with $\omega=0.55$. Defect states are not observed in other gaps for this defect configuration. The mode intensity for this mode is shown in Fig. 5. Due to the change in the local environment of the missing cylinder at site $B$ (Fig. 1), the number of defect modes, their frequencies and the spatial distribution of the defect modes are quite different from those of the defect states at site $A$. We note that removing the central cylinder $(A)$ can induce a defect state in the first gap while removing a cylinder $(B)$ surrounding the central cylinder failed to do so. It can be rationalized by the fact that the effective microcavity formed by removing cylinder $\mathrm{A}$ is larger in size than that of cylinder $B$, and hence site $A$ can accommodate a defect mode of a lower frequency. We now remove a cylinder at site $C$, again resulting in another set of defect states. From the DOS shown in Fig. 6, we observe that defect states are formed at $\omega=0.34$ in the first gap, and at $\omega=0.83$ in the fourth gap. The corresponding mode intensities are shown in Fig. 7. It is interesting to note that the defect state in the first gap for the missing cylinder at site $C$ has nearly the same frequency as the defect mode in the first gap at site $A$. However, the field pattern of these two defect modes are rather different. For completeness, we also show defect modes caused by removing cylinders at other sites, such as $D$ and $E$ in Figs. 8 and 9, respectively. For site $D$, we show the mode intensities for a defect mode in the first gap Fig. 10, and for site $E$, we show the mode intensities for defect modes in the second and the third gaps in Fig. 11. In general, the field patterns have more nodes and are more complicated for the defect modes with higher frequencies. Quasiperiodic photonic band-gap systems may be useful since most of the defect modes in 2D systems that are conceived to be useful in applications are cavity modes created by the removal of a cylinder. It is clear from these results that the quasiperiodic structure offers a great variety of possibilities for creating and controlling the number of defect modes as well as the defect mode frequencies and mode intensity distributions.

\section{B. Right-angled waveguides}

Mekis et al. ${ }^{9}$ have demonstrated that a waveguide constructed from a 2D periodic photonic band-gap structure can bend light around sharp corners. A 2D PBG structure with multiple photonic gaps for TM polarized EM waves can be constructed by arranging dielectric rods in a square array and

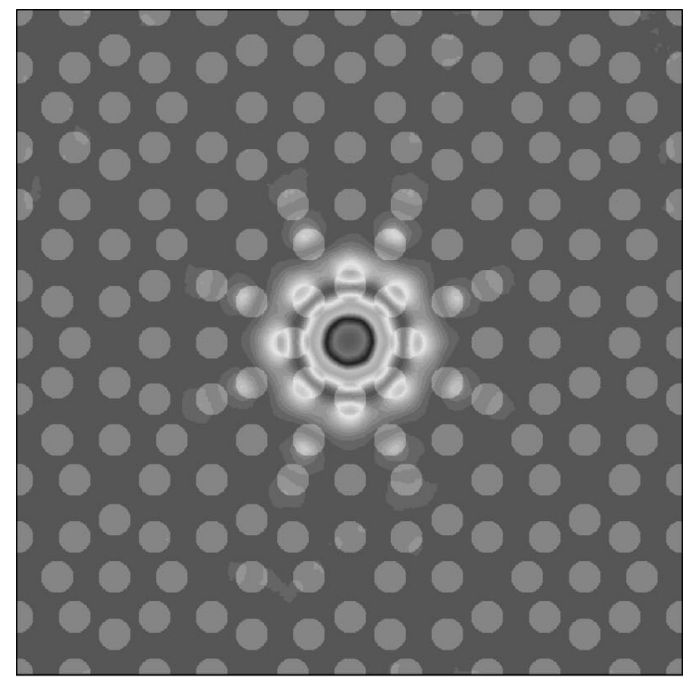

(a)

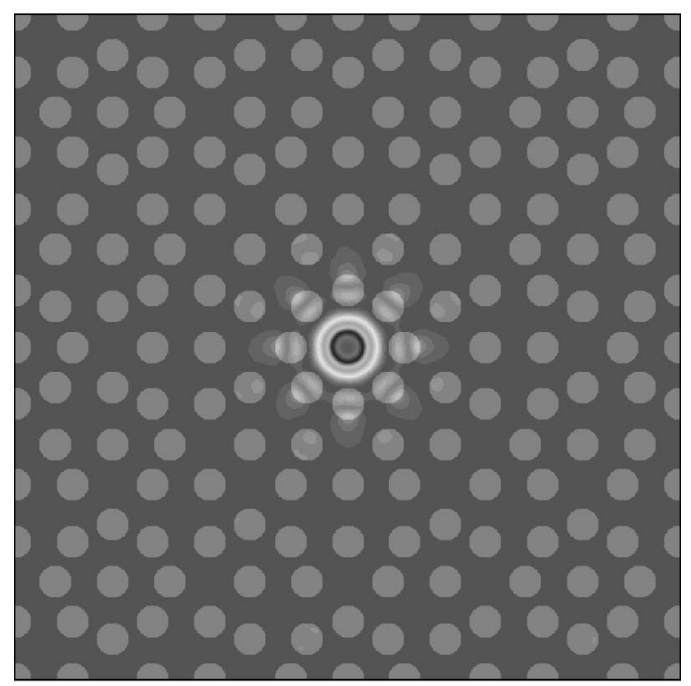

(b)

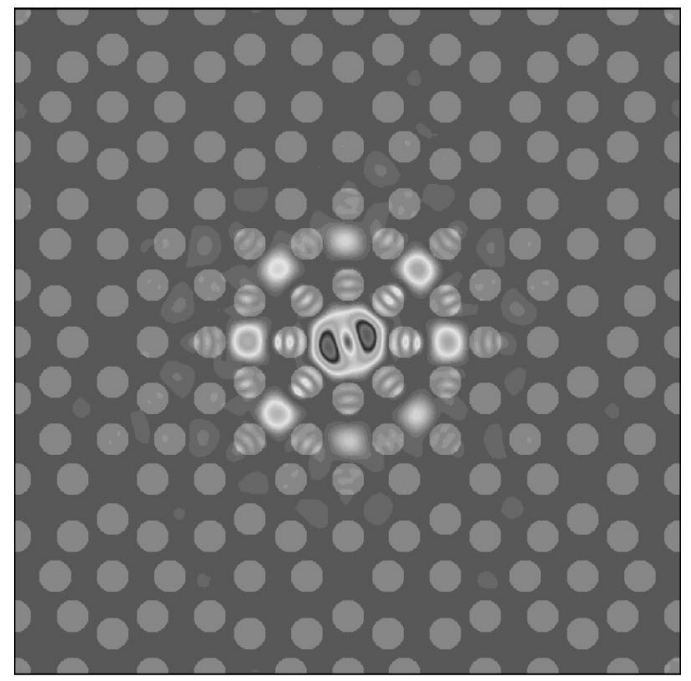

(c)

FIG. 3. 2D projections of the $E$-field intensity of some defect modes for a missing cylinder at position $A$. The top panel is for the defect state with $\omega=0.34$ in the first gap; the middle panel is for the defect state with $\omega=0.51$ in the second gap; and the bottom panel is for $\omega=0.82$ in the fourth gap (see Fig. 1). 


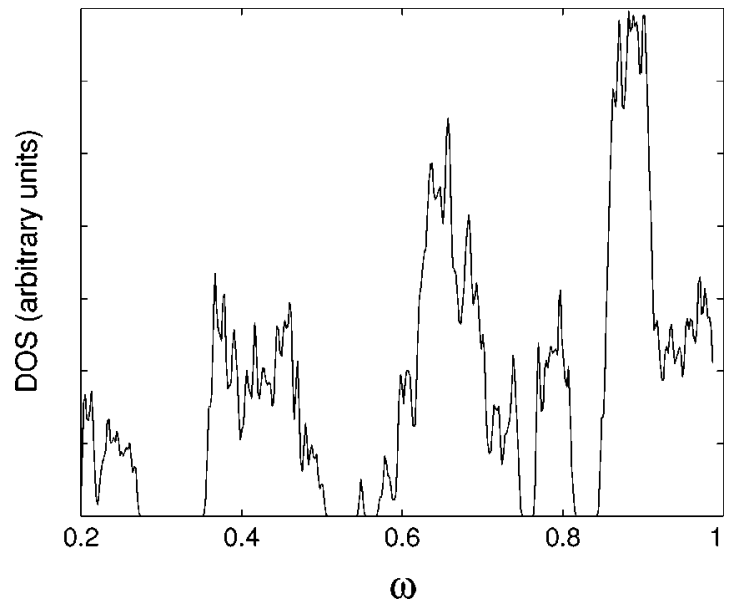

FIG. 4. TM mode density of states for the configuration with a missing cylinder at position $B$, showing a defect mode in the second gap (see Fig. 1).

a waveguide can be formed by removing a row of rods in such a structure. ${ }^{2}$ EM waves with frequencies inside the photonic gaps can only travel inside the channel of missing rods but not inside the bulk of the photonic crystal, and thus the channel of missing rods can serve as a waveguide. When a waveguide formed from removing a row in the $x$ direction terminates abruptly and is joined by another waveguide formed by removing a row in the $y$ direction, a sharp $90^{\circ}$ bend is formed. Normally, light will leak out of an ordinary waveguide (whose function typically depends on the principle of total internal reflection) when it turns abruptly around a corner. However, light cannot leak into the PBG medium if the traveling wave has frequency inside the photonic gap. The incident wave can only be transmitted, or reflected back. A numerical simulation ${ }^{9}$ showed that highly efficient transmission can be obtained for such PBG waveguides with right-angled bends, while the detail of the results depends somewhat on the detail of the structure at the corner of the $90^{\circ}$ bend. We expect that similar behaviors should be observed when we carve waveguides out of qua-

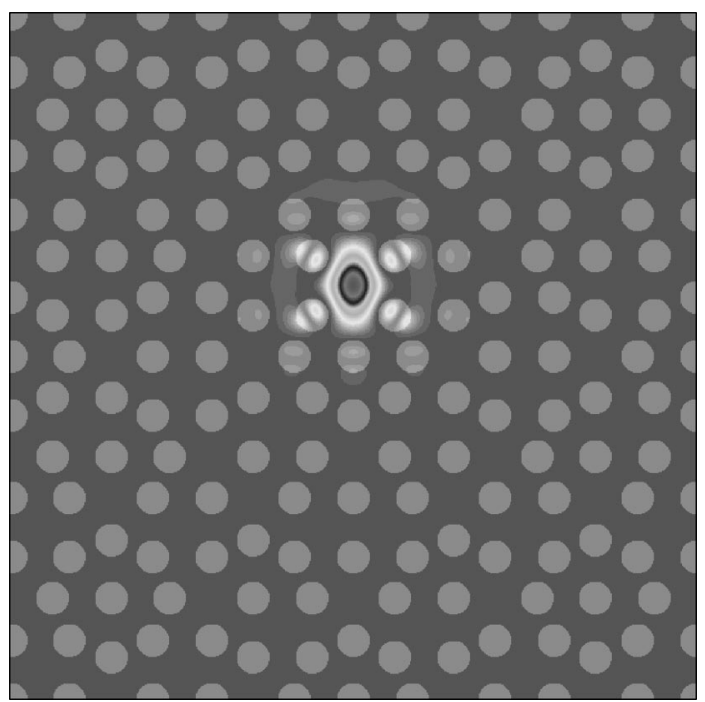

FIG. 5. E-field intensity of the defect mode in the second gap localized near a missing cylinder at position $B$ (see Fig. 1).

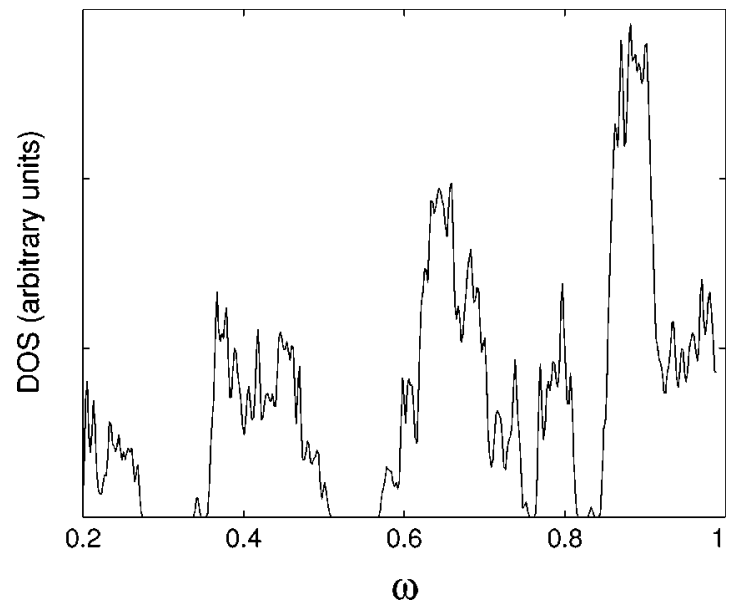

FIG. 6. TM mode density of states for the configuration with a missing cylinder at position $C$ (see Fig. 1).

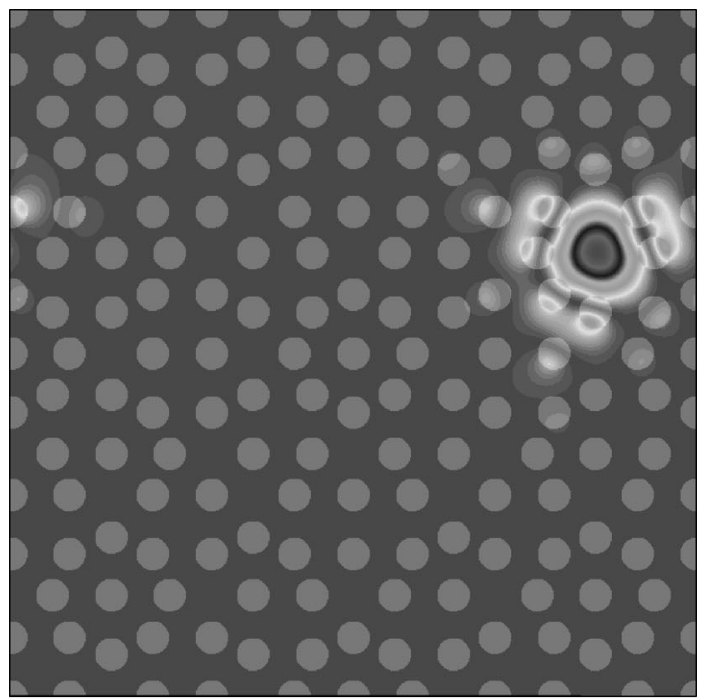

(a)

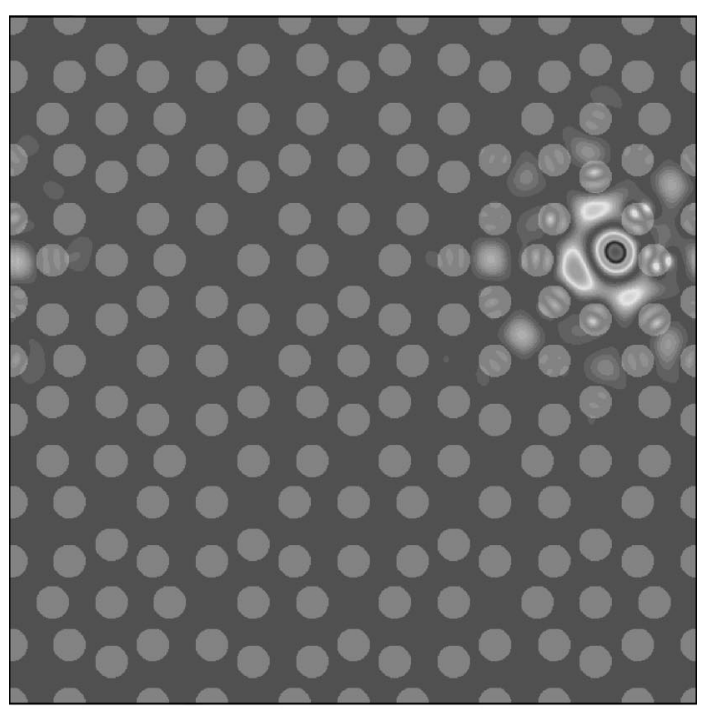

(b)

FIG. 7. E-field intensity of some of the defect modes localized near a missing cylinder at position $C$. The top panel for the state with $\omega=0.34$ and the bottom panel for the state with $\omega=0.83$ (see Fig. 1). 


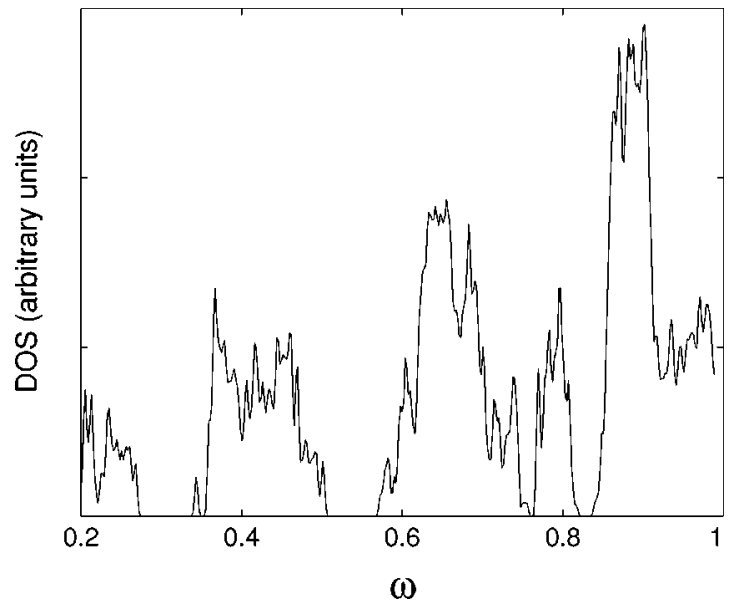

FIG. 8. TM mode density of states for the configuration with a missing cylinder at position $D$ (see Fig. 1).

siperiodic PBG materials. We note that there exists straight rows of cylinders in both the $x$ and $y$ directions in the 2D octagonal photonic quasicrystal, and thus right-angled waveguides with well-defined directions can be formed by removing rows of cylinders. With a finite sample of the octagonal PBG structure, we made such a channel with a rightangled bend which is shown schematically in Fig. 12, where two missing rows of rods intersect at a site which is surrounded by eight nearest-neighbor rods (site A in Fig. 1). In order to investigate the transmission property of such a configuration, we probe the system with a continuous wave with a fixed frequency $\omega$. The s-polarized (TM) wave enters the waveguide at the opening at position $A$, and we calculate the transmitted power at positions $C$ as shown in Fig. 13. For reference, we also show the forward transmitted power in the far field as dotted lines in Fig. 13. For an easier comparison, we have magnified the forward transmitted power by a factor of three. The source of the probe beam is a slit ${ }^{13}$ of width $4 a$ positioned at a distance $6 a$ away from the sample. In this case, the incident field can be obtained from the Kirchoff integral formula. In two dimensions, for a plane wave, $\exp \left(i k_{0} x\right)$, incident from $x<0$, the diffracted wave in the region $x>0$ arising from a slit centered at origin with an opening of width $w$ in the $y$ direction is given by

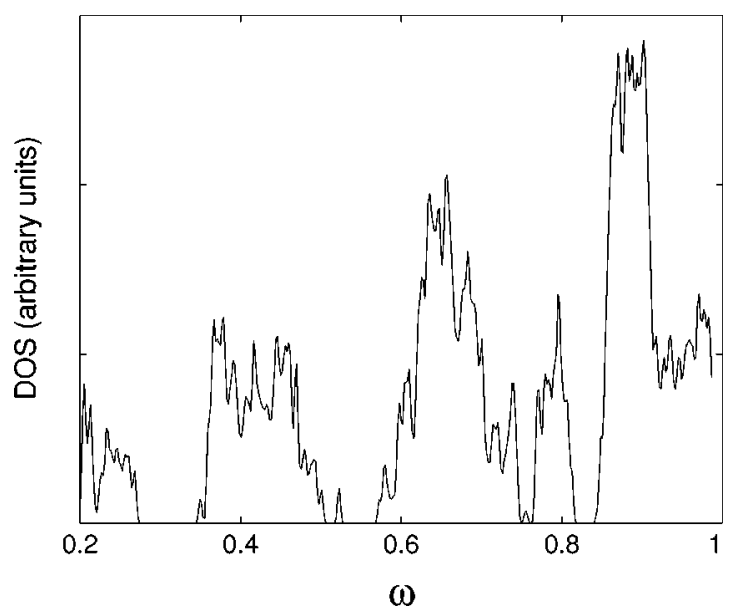

FIG. 9. TM mode density of states for the configuration with a missing cylinder at position $E$ (see Fig. 1).

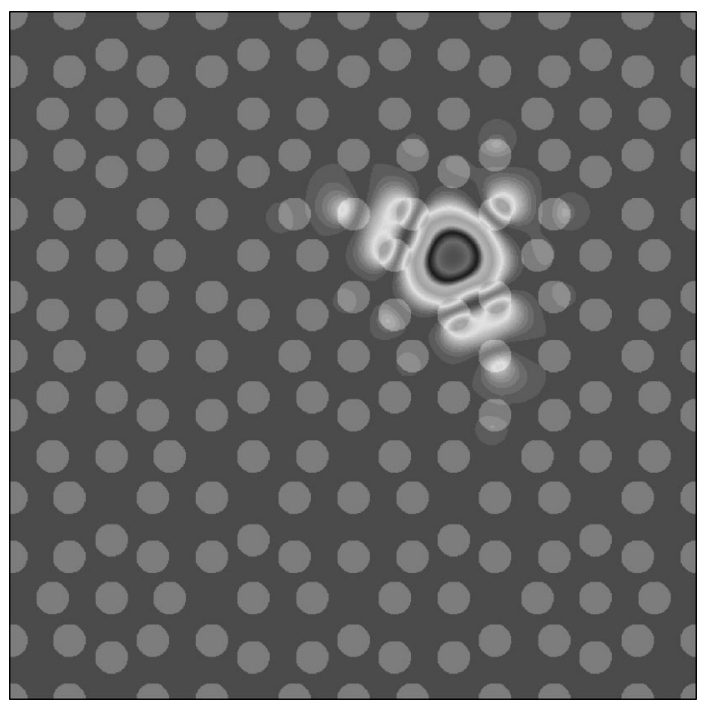

FIG. 10. E-field intensity of a defect mode in the first gap localized near a missing cylinder at position $D$ (see Fig. 1).

$$
u_{\mathrm{inc}}(x, y)=\left(\frac{k_{0}}{4}\right) \int_{-w / 2}^{w / 2} d y^{\prime}\left[H_{0}\left(k_{0} \rho^{\prime}\right)+i \frac{x}{\rho^{\prime}} H_{1}\left(k_{0} \rho^{\prime}\right)\right] \text {, }
$$

where $\rho^{\prime}=\sqrt{x^{2}+\left(y-y^{\prime}\right)^{2}}$, and $H_{m}$ is the Hankel function of the first kind. The Poynting vector is given by

$$
\vec{S}(\vec{\rho})=\frac{-c}{8 \pi k_{0}} \operatorname{Im}[u(\vec{\rho}) \nabla u *(\vec{\rho})],
$$

where $u(\vec{\rho})$ is the value of $E_{z}$ at position $\vec{\rho}$ in the 2D plane. The fields $u(\vec{\rho})$ are calculated by a multiple-scattering formalism. ${ }^{13}$ The transmitted power at position $C$ can be obtained by integrating the Poynting vector of the fields exiting from the aperture at that position and normalized by the integrated power at the aperture at position $A$, where the EM wave enters the channel. We first focus on the configuration given in Fig. 12(a). The dotted line in Fig. 13(a) is the transmitted power for a range of frequencies measured in the forward direction for this configuration. If the system does not have a spectral gap at a particular frequency, at least part of the incident wave can penetrate straight through the PBG material and will be detected in the forward direction. Therefore, the absence of the forward transmitted power signals a stop band for that incident direction. ${ }^{20} \mathrm{We}$ will now focus on the stop band frequencies (where the dotted lines are zero) and look at the power measured at $C$, which represents transmitted power around the corner. We see that there is nearly no power being transmitted by the right-angled waveguide in first stop band near $\omega=0.3$. A rough estimate, treating the opening of the channel as a simple metallic waveguide, shows that the lowest frequency mode allowed in the waveguide is higher than the maximum frequency in the first gap. (There is transmittance for frequencies lower than the first stop band due to diffraction of the beam through the sample.) We will consider the case of a wider channel later but for the moment, we examine the transmission for frequencies in the second stop band, at about $\omega=0.55$. Here, the incident wave has shorter wavelengths and there is clearly transmittance in the spectral gap measured at $C$ as the EM wave is guided 


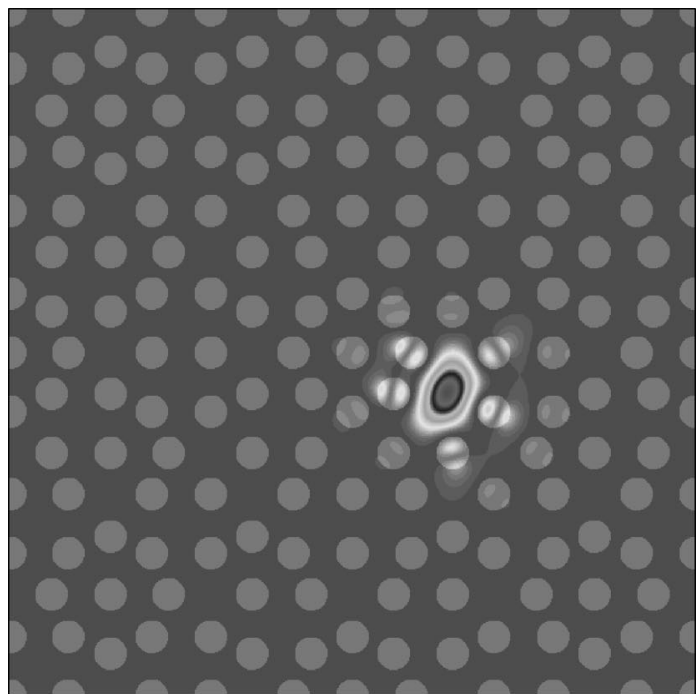

(a)

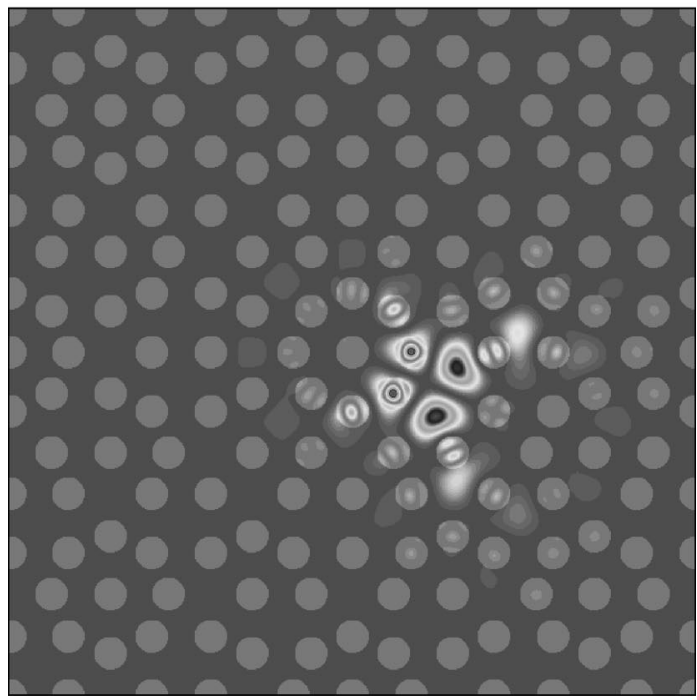

(b)

FIG. 11. E-field intensity of some defect modes localized near the missing cylinder at position $E$. The top panel is for the defect state in the second gap $(\omega=0.53)$, and the bottom panel is for the state in the third gap $(\omega=0.76)$ (see Fig. 1).

through the channel and around the sharp corner. The behavior is somewhat different from that of the waveguide channels crafted out of periodic PBG systems, where the transmission efficiency is very high for nearly all frequencies inside the gap. ${ }^{9}$ The transmission spectrum for quasiperiodic structure is rather rich in structure and only some frequencies get near perfect transmission. This difference is due to the property of quasiperiodicity of the host lattice. It has been demonstrated that in one-dimensional quasiperiodic photonic band-gap systems (stacks of two different materials arranged in a Fibonacci sequence), the transmittance spectrum is highly structured. ${ }^{21}$ Our PBG waveguide is formed by removing material from a quasiperiodic system, and the resulting structure is an one-dimensional channel bounded by a wall of quasiperiodic modulation. Since the channel formed by removing one row of cylinders is narrow, the effect of the boundary is rather significant and we thus see a highly structured transmission spectrum with high transmittance only for
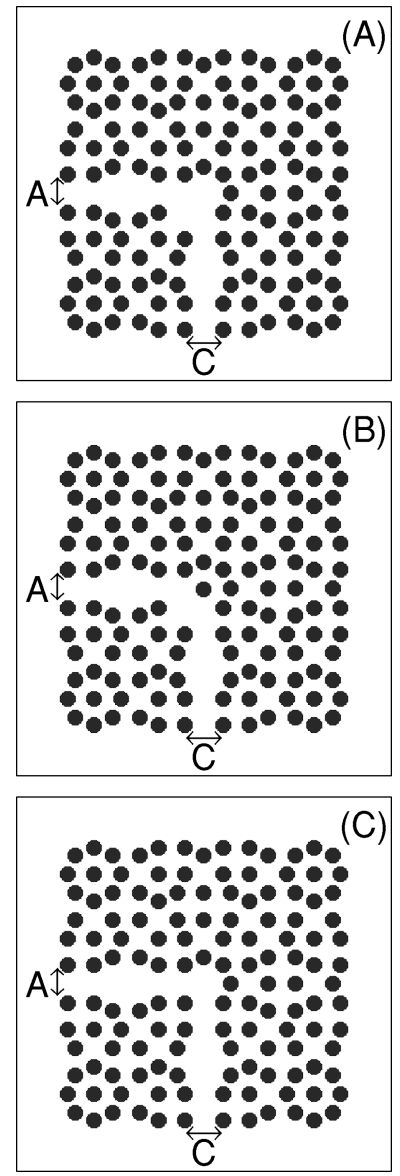

FIG. 12. Schematic figure showing right-angled waveguides formed from removing a row of cylinders in a quasiperiodic PBG system. Panels (A), (B), and (C) differ only in the arrangement of cylinders at the bend. A continuous wave of angular frequency $\omega$ enters the system at aperture $A$, and the transmitted power is monitored at position $C$.

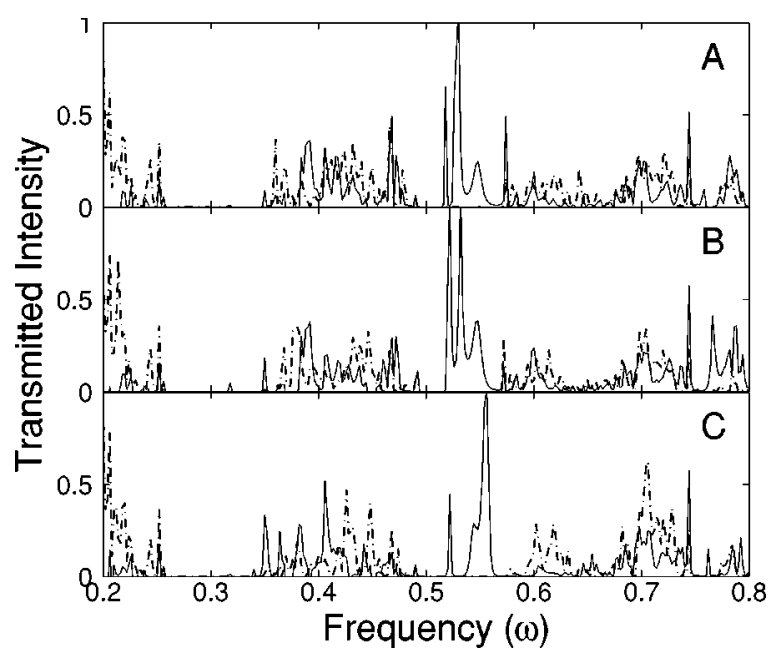

FIG. 13. The transmitted power measured in the forward direction (dotted line) and at position $C$ (solid lines) corresponding to the three configurations depicted in Fig. 12. The transmitted power measured in the forward direction (dotted line) has been magnified three times. The absence of forward transmitted power through the PBG material indicates stop bands. 


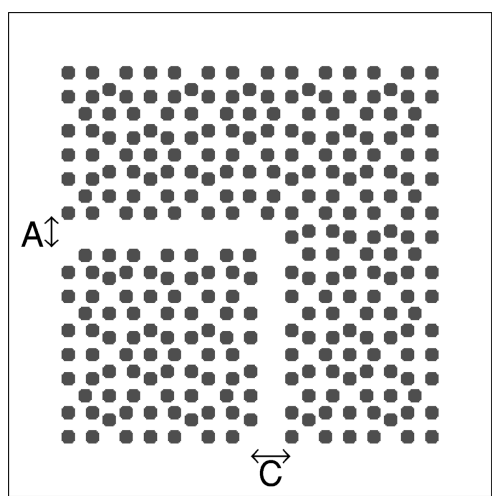

FIG. 14. Schematic figure showing another right-angled waveguide configuration formed from removing two rows of cylinders in a quasiperiodic PBG system. A continuous wave of angular frequency $\omega$ enters the system at aperture $A$, and the transmitted power is monitored at $C$.

some frequencies inside the frequency window set by the spectral gap. The $90^{\circ}$ bend basically serves as a coupler between two channels in different directions and thus the arrangement of cylinders at the bend is important. This point is illustrated by comparing the transmission properties of a few right-angle waveguides as depicted in Fig. 12. Only the structure at the corner is different, and we can see from the corresponding calculated transmission spectra (Fig. 13) that the frequencies with high transmittance are not the same. The bend apparently "picks" some particular frequencies to transmit.

As in the case of defects, the quasiperiodic arrangement of cylinders offer many different possibilities in crafting waveguides. In Fig. 14, we show another waveguide configuration created by removing cylinders resulting in a slightly wider channel. In contrast to the configurations shown in Fig. 12, which have wavy boundaries, this configuration has straight boundaries. The corresponding transmission spectrum is shown in Fig. 15. Here again, the dotted

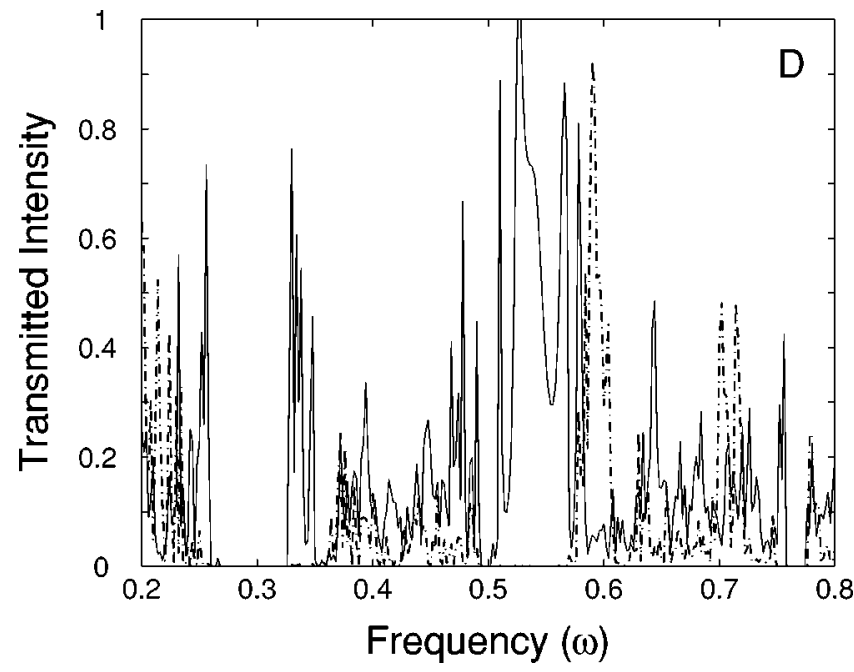

FIG. 15. Transmitted power measured in the forward direction (dotted line) and at $C$ (solid lines) corresponding to the situation depicted in Fig. 14. The transmitted powers measured in the forward direction (dotted line) through the PBG material have been magnified three times for an easier comparison.

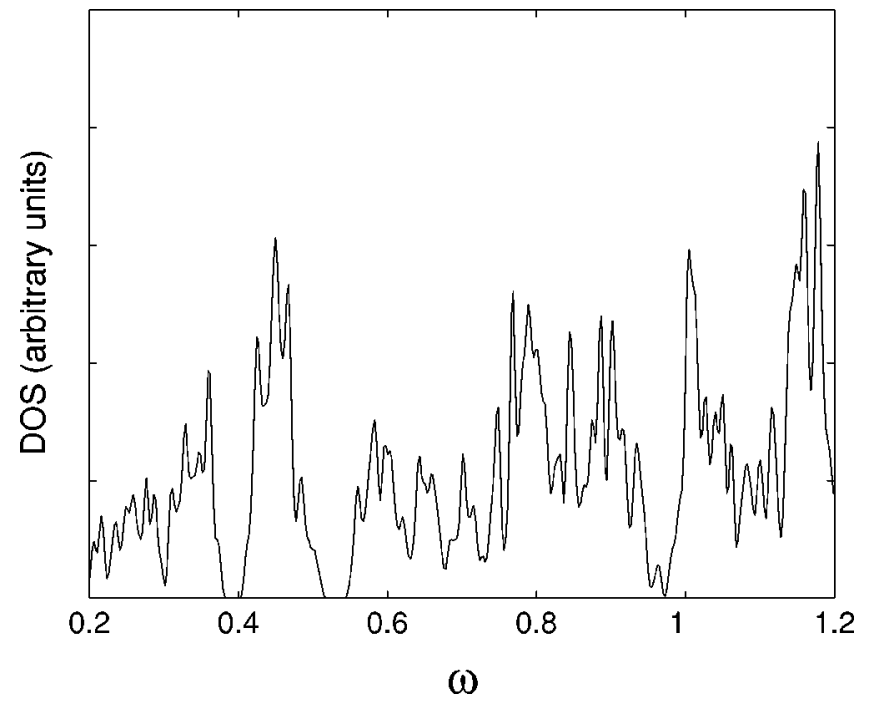

FIG. 16. TE mode DOS for the octagonal quasiperiodic metallic cylinder array.

lines are the transmitted power passing through the PBG system in the forward direction (magnified three times) and an absence of transmitted power through the system indicates the existence of a spectral gap in that direction. The solid line is the transmitted power that goes through the right-angled bend. We now see some transmission at position $C$ for frequencies the first gap. This is because the channel is now wider. Compared with the configurations depicted in Fig. 12, this wider channel with straighter boundary is less selective in frequency but the transmission spectrum is still rather spiky. We expect that the difference between the transmission characteristics for periodic and quasiperiodic PBG waveguides will fade away as the channel grows wider.

From these results, we see that quasiperiodic PBG material can also guide light around sharp corners, but it is more frequency selective as compared with periodic PBG. There is then a possibility that the quasiperiodic PBG system can serve as a light guide with filtering effect.

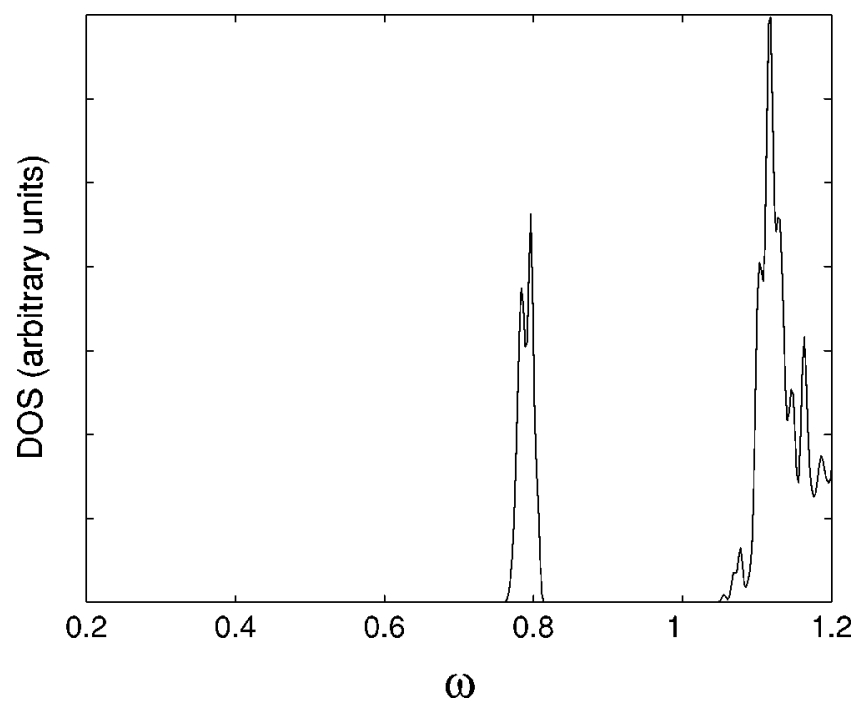

FIG. 17. TM mode DOS for the octagonal quasiperiodic metallic cylinder array. 


\section{Metallodielectric configurations}

The structure shown in Fig. 1, which consists of isolated high dielectric cylinders in a lower dielectric background, has spectral gaps for TM modes. Such structures do not have complete photonic gaps for the TE modes. We found that spectral gaps for TE modes require a connected structure of high dielectrics and can be realized in a connected vein structure as shown in Fig. 5 of Ref. 11. Although the two polarizations are decoupled in two dimensions, it would be nice if we can find a structure that supports photonic gaps simultaneously for both polarizations in the same frequency range. We found that there is at least one such possibility, if we use metallodielectric elements. Such a system can be realized by replacing all the dielectric rods in Fig. 1 by ideal metallic cylinders. The metallic cylinders are chosen to have radii of $0.3 a$. The perfect metal is modeled by setting all the $E$ fields to zero in each of the time steps in the simulation, and physically it means that the metallic domain corresponds to a region that repels fields. The simulations are done for systems containing 478 cylinders, with a $2000 \times 2000$ grid. The DOS for the TE modes and the TM modes are shown in Figs. 16 and 17, respectively. The TE mode DOS (Fig. 16) exhibit spectral gaps at about $\omega=0.39$ and 0.53 . The frequency ranges of these gaps are also forbidden in the TM modes, as can be observed in Fig. 17. It is possible that for a system with more cylinders, a small gap can open at about $\omega$ $=0.95,{ }^{22}$ which is also forbidden in the TM mode. Although this system of isolated metallic cylinders embedded in a dielectric background has the same geometrical arrangement as the isolated dielectric cylinder arrangement shown in Fig. 1, there are differences in the way they give rise to photonic gaps. The metallic component excludes fields, so that the EM wave in this case actually passes through the material in the dielectric background, which forms a percolated structure having the symmetry of an octagonal quasiperiodic lattice. In a way, the metallic cylinder configuration is quite similar to the connected vein structure (Fig. 5 of Ref. 11) that supports TE mode photonic gaps. For the TM mode, the $E$ field is parallel to the axis of the metallic cylinders and the $E$ field has to be zero on the surface of the cylinder. It is expected that em waves with wavelength less than the typical separation between the cylinders cannot pass through the medium, ${ }^{23}$ and hence there is a gap at the lower frequencies. By examining the field intensity plots, we found that the group of states near $\omega=0.8$ are highly localized about some sites in the PBG systems, while the states above $\omega=1$ look delocalized. Metallodielectric structures as PBG materials for different applications have been advocated by a few groups. ${ }^{23,24}$ This type of metallodielectric structure may have its own disadvantage because of possible strong dissipation effects of the metallic elements, depending on the operating frequency range. On the other hand, they have some advantage since we can easily control the position of the gaps by varying the dielectric constant of the dielectric background material. For example, the frequency of the gaps basically scales inversely as $\sqrt{\epsilon}$ of the background material. We can thus have spectral gaps at lower frequencies by employing a higher dielectric background material without changing the physical dimensions of the photonic quasicrystal.

\section{SUMMARY}

Point defects (missing cylinders) and line defects (missing rows of cylinders) can be used to create highly localized defect modes and to form waveguides in photonic band-gap systems. These effects depend on the rejection of EM waves in the photonic band gap and as long as quasiperiodic PBG systems have photonic gaps, we should see these effects as in the case of periodic PBG systems. However, the intrinsic difference between aperiodic and periodic systems, such as the absence of translational symmetry and the difference in wave propagation characteristics, lead to some differences. For example, the defect properties become much richer and the "light guide", derived from aperiodic PBG structures becomes more frequency selective. These extra complexities may be exploited in some situations to provide better tunability in certain PBG applications. Spectral gaps common for both the TM and TE polarizations can be achieved in metallodielectric quasiperiodic photonic band-gap system. We are still searching for aperiodic structures that support spectral gaps for both polarizations in the same frequency range with dielectric material only.

\section{ACKNOWLEDGMENT}

We thank CCST-HKUST for computer time on the Paragon. S.C. and C.T.C. were supported by RGC Hong Kong through HKUST6136/97P. L.M.L. and Z.Q.Z. were supported by HKUST6137/97P.
${ }^{1}$ See, for example, Photonic Band Gaps and Localization, edited by C.M. Soukoulis (Plenum, New York, 1993); J. Opt. Soc. Am. B 10, 208-408 (1993); Photonic Band Gap Materials, NATO ASI Series, edited by C.M. Soukoulis (Kluwer, Dordrecht, 1996).

${ }^{2}$ J. Joannopoulos, R.D. Meade, and J. Winn, Photonic Crystals (Princeton University, Princeton, NJ, 1995).

${ }^{3}$ E. Yablonovitch, Phys. Rev. Lett. 58, 2059 (1987).

${ }^{4}$ See, e.g., S. John and T. Quang, Phys. Rev. Lett. 74, 3419 (1995); 76, 1320 (1996); S. Y. Zhu, H. Chen, and H. Huang, ibid. 79, 205 (1997).

${ }^{5}$ S. John, Phys. Rev. Lett. 58, 2486 (1987).
${ }^{6}$ T.F. Krauss, D. Labilloy, A. Scherer, and R.M. De La Rue, Proc. SPIE 3278, 306 (1998).

${ }^{7}$ R.D. Meade, A. Devenyi, J.D. Joannopoulos, O.L. Alerhand, D.A. Smith, and K. Kash, J. Appl. Phys. 75, 4753 (1994).

${ }^{8}$ K. Agi, E.R. Brown, D.B. McMahon, C. Dill, and K.J. Malloy, Electron. Lett. 30, 2166 (1994); M.M. Sigalas, R. Biswas, Q. Li, D. Crouch, W. Leung, R. Jacobs-Woodbury, B. Laogh, S. Nielsen, S. McCalmont, G. Tuttle, and K.M. Ho, Microwave Opt. Technol. Lett. 15, 153 (1997).

${ }^{9}$ A. Mekis et al., Phys. Rev. Lett. 77, 3787 (1996).

${ }^{10}$ See, e.g., K.M. Leung, Phys. Rev. B 56, 3517 (1997); C.M. Anderson and K.P. Giapis, Phys. Rev. Lett. 77, 2949 (1996). 
${ }^{11}$ Y.S. Chan, C.T. Chan, and Z.Y. Liu, Phys. Rev. Lett. 80, 956 (1998).

${ }^{12}$ C.T. Chan, Q.L. Yu, and K.M. Ho, Phys. Rev. B 51, 16635 (1995).

${ }^{13}$ L.M. Li and Z.Q. Zhang, Phys. Rev. B 58, 9587 (1998).

${ }^{14}$ See, e.g., K.M. Leung and Y.F. Liu, Phys. Rev. Lett. 65, 2646 (1990); Z. Zhang and S. Satpathy, ibid. 65, 2650 (1990); K.M. Ho, C.T. Chan, and C.M. Soukoulis, ibid. 65, 3152 (1990); C.T. Chan, K.M. Ho, and C.M. Soukoulis, Mod. Phys. Lett. B 6, 139 (1992).

${ }^{15}$ K.S. Yee, IEEE Trans. Antennas Propag. AP-14, 302 (1966).

${ }^{16}$ G. Tayeb and M. Maystre, J. Opt. Soc. Am. A 14, 3323 (1997).

${ }^{17}$ See, e.g., H. C. van de Hulst, Light Scattering by Small Particles (Dover, New York, 1981).

${ }^{18}$ See, e.g., Z.Y. Liu, Q. Tiang, Z. Zhang, and D. Tian, J. Phys.: Condens. Matter 4, 6343 (1992).

${ }^{19}$ In Ref. 11 , the frequency units are given as $2 \pi(c / l)$ where $l$ is the length of a square simulation cell used to calculate the photon DOS. The square cell that was chosen has $l=(6+4 \sqrt{2}) a$, where $a$ is the length of a side of the square (or rhombuses) in the octagonal tiling. In this article, all frequency units are taken to be $2 \pi(c / a)$. Thus the frequencies quoted in Ref. 11 should be divided by 11.657 for a direct comparison with the numbers quoted here.
${ }^{20}$ Strictly speaking, the absence of transmission gives only the stop band in one direction, while a zero density of states indicates a bona fide spectral gap (in all directions).

${ }^{21}$ See, e.g., M. Kohmoto, B. Sutherland, and K. Iguchi, Phys. Rev. Lett. 58, 2436 (1987); T. Hattori, N. Tsurumachi, S. Kawato, and H. Nakatsuka, Phys. Rev. B 50, 4220 (1994).

${ }^{22}$ By varying the size of the sample, we found in general that larger samples have more spiky DOS. While the main spectral gaps remain stable and quickly converge with the sample size, more gaps are opened as the size of the sample increases. Although the TE mode gaps at $\omega=0.39$ and 0.53 are small, they are very robust with respect to the size of sample varying from 164 cylinders to 478 cylinders. The DOS results shown for Figs. 16 and 17 correspond to a realization of the octagonal quasiperiodic arrangement using 478 cylinders. It is likely that a larger sample will have a gap for TE modes at about $\omega=0.95$. We cannot afford to test larger samples due to computation resources limitation.

${ }^{23}$ M.M. Sigalas, C.T. Chan, K.M. Ho, and C.M. Soukoulis, Phys. Rev. B 52, 11744 (1995).

${ }^{24}$ See, e.g., K.A. McIntosh, O.B. McMahon, and S. Verghese, Microwave Opt. Technol. Lett. 17, 153 (1998); D.F. Sievenpiper, E. Yablonovitch, J.M. Winn, S. Fan, P.R. Villeneuve, and J.D. Joannopoulos, Phys. Rev. Lett. 80, 2829 (1998). 